\title{
Uptake of conjugated linolenic acids and conversion to cis-9, trans-11-or trans-9, trans-11-conjugated linoleic acids in Caco-2 cells
}

\author{
Anne-Catherine Schneider ${ }^{1,2}$, Eric Mignolet ${ }^{1,3}$, Yves-Jacques Schneider ${ }^{1,2}$ and Yvan Larondelle ${ }^{1 *}$ \\ ${ }^{1}$ Institut des Sciences de la Vie, Université catholique de Louvain, Croix du Sud, 2, bte L7·05.08, B-1348 Louvain-La-Neuve, \\ Belgium \\ ${ }^{2}$ Croix du sud, 4-5, bte L7.07.03, B-1348 Louvain-La-Neuve, Belgium \\ ${ }^{3}$ Croix du sud, 2, bte L7·05.08, B-1348 Louvain-La-Neuve, Belgium \\ (Submitted 1 September 2011 - Final revision received 30 November 2011 - Accepted 27 January 2012 - First published online 4 April 2012)
}

\section{Abstract}

Dietary oils containing large amounts of conjugated linolenic acids (CLnA) may be regarded as a source of conjugated linoleic acids (CLA), which have been suspected to bear health-promoting properties. Indeed, CLnA can be converted into CLA in mammals. The objective of the present study was to investigate the uptake of CLnA and their metabolism into CLA in Caco- 2 cells, as a validated in vitro model of the intestinal barrier. Caco- 2 cells were incubated for $24 \mathrm{~h}$ in the presence of either $\alpha$-eleostearic, $\beta$-eleostearic, catalpic or punicic acid. We first observed that Caco-2 cells take these CLnA up at different rates and then convert them but with varying efficiency depending on the structure of the $\Delta 13$ double bond. Finally, the distribution of CLnA between neutral lipids (NL) and phospholipids appeared to be linked to their number of trans double bonds: the higher the number, the higher the accumulation in the NL fraction.

Key words: $\boldsymbol{\alpha}$-Eleostearic acid: $\beta$-Eleostearic acid: Catalpic acid: Punicic acid: Conjugated linolenic acids: Conjugated linoleic acids: Caco-2 cells

Conjugated fatty acids refer to a group of positional and geometric isomers of PUFA with conjugated double bonds. The most studied are conjugated linoleic acids (CLA), with two conjugated double bonds, and conjugated linolenic acids (CLnA), with three conjugated double bonds ${ }^{(1-3)}$ (Fig. 1). It has been established in vitro and in vivo by several authors that CLA, and more specifically cis-9, trans-11 (c9t11)-CLA and/or $t 10 c 12$-CLA, have a health-promoting potential regarding the prevention against some cancers ${ }^{(4,5)}$ and atherosclerosis $^{(6,7)}$, as well as the attenuation of inflammatory processes $^{(8-10)}$. CLA occur naturally and are mainly present in products derived from ruminants, such as milk fat, cheese and meat. The principal dietary form is c9t11-CLA, also named rumenic $\operatorname{acid}^{(2,8,11)}$. However, the CLA level in ruminant products ranges between 2 and $7 \mathrm{mg} / \mathrm{g}$ fat ${ }^{(12)}$, which represents thus less than $1 \%$ of total lipids, maintaining therefore the intake in CLA from dietary products relatively low. In contrast to this low content in CLA, levels in CLnA in seed oil of certain plants can reach up to $70-80 \%$ of the total lipids. More specifically, $c 9 t 11 c 13-C L n A$ (punicic acid, PA) was found at up to $83 \%$ in pomegranate seed oil (Punicia granatum), whereas $c 9 t 11 t 13$-CLnA ( $\alpha$-eleostearic acid, $\alpha$-ESA) was recovered at up to $67 \cdot 7 \%$ and $56.2 \%$ in tung seed oil and bitter gourd seed oil (Momordica charantia), respectively, and $t 9 t 11 c 13-C L n A$ (catalpic acid, CA) at up to $42 \cdot 3 \%$ in catalpa seed oil (Catalpa ovata) ${ }^{(13)}$. In contrast to these three natural isomers, the fourth isomer that was used in this study, namely the $19 t 11 t 13$-CLnA ( $\beta$-eleostearic acid, $\beta$-ESA), results mainly from chemical synthesis ${ }^{(14)}$.

CLnA isomers share the conjugated double bond system of CLA, as well as the carbon structure (18:3) of $\alpha$-linolenic acid, conferring to these fatty acids some beneficial effects on health ${ }^{(14)}$. In mammals, it has been reported that some isomers of $-9,-11,-13$ CLnA could improve the immune response $^{(15,16)}$ and parameters linked to obesity ${ }^{(17,18)}$ or have anti-carcinogenic effects ${ }^{(19-23)}$. These properties are closely related to those of CLA and it cannot be excluded that some of them are likely to be related to a relatively important conversion of $-9,-11,-13$ CLnA into $c 9 t 11$ or $t 9 t 11-C L A$. Indeed, Tsuzuki et $a l^{(24,25)}$ and Yuan et al. ${ }^{(26-28)}$ have previously reported that $\alpha$-ESA and PA can be metabolised into $c 9 t 11$ CLA in rats, mice and humans. Suzuki et al. ${ }^{(20)}$ also showed

Abbreviations: $\alpha$-ESA, $\alpha$-eleostearic acid; $\beta$-ESA, $\beta$-eleostearic acid; CA, catalpic acid; CLA, conjugated linoleic acids; CLnA, conjugated linolenic acids; FAME, fatty acid methyl esters; LTB4 12-HD, leukotriene B4 12-hydroxydehydrogenase; NL, neutral lipids; PA, punicic acid; PGR, 15-ketoPG13-reductase; PL, phospholipids.

*Corresponding author: Dr Y. Larondelle, fax +32 10473728, email yvan.larondelle@uclouvain.be 


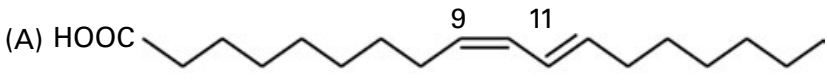

(B) $\mathrm{HOOC}$
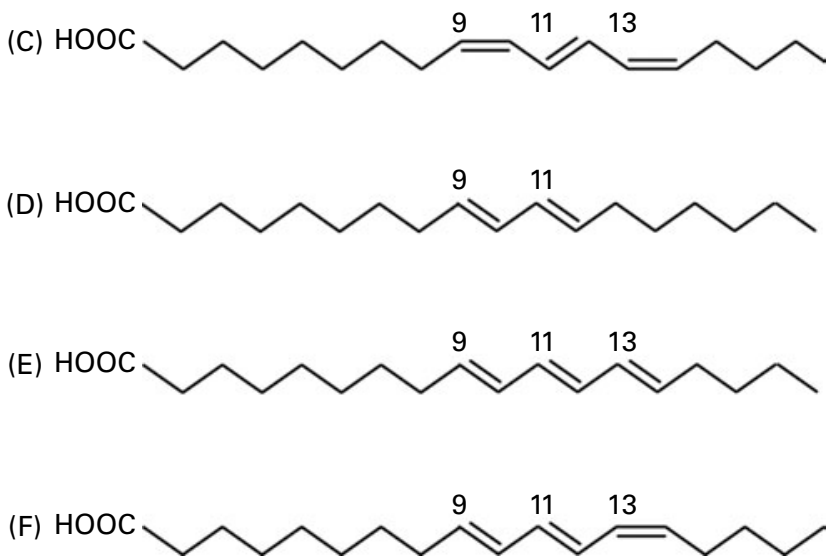

Fig. 1. Structures of (A) cis-9, trans-11-conjugated linoleic acids (CLA), (B) $\alpha$-eleostearic acid, (C) punicic acid, (D) trans-9, trans-11-CLA, (E) $\beta$-eleostearic acid and (F) catalpic acid.

that oral administration of catalpa seed oil, containing more than $40 \%$ of CA, to rats, induced the accumulation of $t 9 t 11$ CLA in the liver and colonic mucosa. The mechanism whereby isomers of $-9,-11,-13$ CLnA are converted into CLA remains unclear. Nevertheless, some authors suggest a mechanism, which implies that the $\Delta 13$ double bond of these CLnA is reduced by an NADPH-dependent enzyme. Tsuzuki et $a$. $^{(24,25)}$ suggest that this conversion is carried out by either a novel enzyme recognising conjugated trienoic acids or the enzyme reducing leukotriene B4, i.e. the leukotriene B4 12-hydroxydehydrogenase (LTB4 12-HD), also named 15-ketoPG13-reductase (PGR). LTB4 12-HD/PGR is a bifunctional enzyme capable of inactivating leukotriene $\mathrm{B} 4$ and $15-\mathrm{OxO}-\mathrm{PG}^{(29)}$. To our knowledge, the conversion of these four CLnA was never reported in the same model.

The intestinal mucosa is the first body structure in contact with dietary CLnA. More specifically, the enterocytes present in the gastro-intestinal tract are of particular interest since they form the site for fatty acid absorption ${ }^{(30,31)}$. Furthermore, these cells are also able to metabolise fatty acids ${ }^{(32)}$. The present study is therefore focused on the uptake of four $-9,-11,-13$ isomers of CLnA, as well as their potential metabolism into CLA in the human intestinal cell line Caco-2, cultivated in serum-free conditions. These cells, widely used, are indeed able to take up and to metabolise extracellular fatty $\operatorname{acids}^{(33-35)}$. In addition, we have previously developed culture conditions that allow the cultivation of $\mathrm{CacO}-2$ cells in a serum-free medium ${ }^{(36,37)}$, allowing easy modulation of the fatty acid composition of the culture medium used. This serum-free medium permits avoiding of the inhibiting effect of some PUFA on the enzymes of lipid metabolism and also permits a better efficiency of the conversion of some 18-carbon fatty acids (personal data). In this study, we aimed at evaluating to which extent these cells can take up and metabolise the four CLnA differing only by the configuration of their double bonds.

\section{Materials and methods}

\section{Cell culture}

Caco-2 cells were purchased from the American Type Culture Collection (ATCC) and were used between passages 42 and 49. Cells were cultured in a serum-free hormono-defined synthetic medium (Basal Defined Medium ${ }^{(36,37)}$ ). Basal Defined Medium was composed of a 5:5:1 (by vol.) mixture of Iscove's modified Dulbecco's medium (Lonza), Ham's F12 medium (Lonza) and NCTC-135 (Invitrogen) supplemented with 1\% (v:v) non-essential amino acids (Lonza), 2 mm-L-glutamine (Lonza), 0.06 mm-ethanolamine (Sigma-Aldrich), 1 $\mu \mathrm{g} / 1$ epidermal growth factor (Sigma-Aldrich), $100 \mathrm{nmol} / 1$ hydrocortisone (Sigma-Aldrich), $2 \mathrm{nmol} / \mathrm{l}$ triiodothyrosin (Sigma-Aldrich), $1 \mathrm{mg} / 1$ insulin (Sigma-Aldrich), $10 \mathrm{mg} / 1$ albumin complexed to linoleic acid (Sigma-Aldrich) and $3 \mathrm{~g} / 1 \mathrm{NaHCO}_{3}$ (SigmaAldrich). Caco-2 cells were maintained at $37^{\circ} \mathrm{C}$ in a humidified atmosphere made of $5 \% \mathrm{CO}_{2}(\mathrm{v}: \mathrm{v})$ in air and were seeded on type I collagen ( $1 \%$ in PBS, v:v) (Sigma-Aldrich) precoated supports. Cells were sub-cultured after reaching 90\% confluence by a HyQ-Tase solution (Perbio-Sciences, HyClone). After sub-cultivation, the cells were seeded at a density of $3 \times 10^{4}$ cells $/ \mathrm{cm}^{2}$ in either six-well plates or $25 \mathrm{~cm}^{2}$ flasks and grown for $9 \mathrm{~d}$ with medium changes every $2 \mathrm{~d}$.

\section{Lipid treatment}

At $7 \mathrm{~d}$ post-confluence ( $9 \mathrm{~d}$ of culture in total), Caco- 2 cells were treated with fatty acids or ethanol vehicle (1\%,v:v; Sigma-Aldrich) for $24 \mathrm{~h}$. All fatty acids ( $\alpha$-ESA, PA, $\beta$-ESA and CA) were purchased from Larodan and dissolved in ethanol. They were added to the cells at a concentration of $20 \mu \mathrm{mol} / \mathrm{l}$, in the presence of $1 \mathrm{~mm}$-taurocholic acid (Sigma-Aldrich).

\section{Fatty acid analysis}

After $24 \mathrm{~h}$ of treatment, the cells were washed, detached and collected in $1 \mathrm{ml}$ of PBS. The cell suspension was then divided in two parts, one for lipid extraction and the other for protein content determination. Both parts were pelleted by centrifugation for $10 \mathrm{~min}$ at $500 \mathrm{~g}$. Total cell lipids were then extracted with chloroform-methanol-water (2:2:1.8; by vol.) (Biosolve) according to the Bligh \& Dyer method ${ }^{(38)}$. An internal standard, tridecanoic acid (C13:0; Sigma-Aldrich), was used in order to quantify the fatty acids present in the lipid extract. Each sample was then dried under nitrogen and methylated at $70^{\circ} \mathrm{C}$ through the addition of $1 \mathrm{ml}$ of $0.1 \mathrm{~mol} / 1 \mathrm{KOH}$ in methanol and a $1 \mathrm{~h}$ incubation followed by the addition of $0.4 \mathrm{ml}$ of $1.2 \mathrm{~mol} / 1 \mathrm{HCl}$ in methanol and incubation during $15 \mathrm{~min}$. The fatty acid methyl esters (FAME) were then extracted using $2 \mathrm{ml}$ of hexane (Biosolve). 
The FAME were separated by GC. The GC Trace (Thermo Finnigan) GC was equipped with an RT2560 capillary column $(100 \mathrm{~m} \times 0.25 \mathrm{~mm}$ internal diameter, $0.2 \mu \mathrm{m}$ film thickness; Restek), a GC PAL autosampler (CTC analystics) and a flame ionisation detector. The carrier gas used was $\mathrm{H}_{2}$ at a constant pressure of $200 \mathrm{kPa}$. The flame ionisation detector was kept at a constant temperature of $255^{\circ} \mathrm{C}$. The temperature program was as follows: an initial temperature of $80^{\circ} \mathrm{C}$, which progressively increased at $25^{\circ} \mathrm{C} / \mathrm{min}$ up to $175^{\circ} \mathrm{C}$; a holding temperature of $175^{\circ} \mathrm{C}$ during $25 \mathrm{~min}$ followed by an increase at $10^{\circ} \mathrm{C} / \mathrm{min}$ up to $205^{\circ} \mathrm{C}$; a holding temperature of $205^{\circ} \mathrm{C}$ during $4 \mathrm{~min}$ followed by a new increase at $10^{\circ} \mathrm{C} / \mathrm{min}$ up to $225^{\circ} \mathrm{C}$ and a holding temperature of $225^{\circ} \mathrm{C}$ during $20 \mathrm{~min}$. A decrease at $20^{\circ} \mathrm{C} / \mathrm{min}$ was then imposed down to the initial temperature of $80^{\circ} \mathrm{C}$. The GC system was connected to a computer with a ChromQuest (version 4.2; Thermo Finnigan) software.

\section{Separation by solid phase extraction}

After extraction (see previous subsection), the total lipid extracts of certain samples were separated by solid phase extraction (Bond Elut- $\mathrm{NH}_{2}, 200 \mathrm{mg}, 3 \mathrm{ml}$; Varian) into three lipid fractions, i.e. neutral lipids (NL), NEFA and phospholipids (PL). A mixture of three internal standards made of triheptadecanoin (Larodan), tridecanoic acid and 1,2-dipentadecanoyl-sn-glycero-3-phosphatidylcholine (Larodan) was added to each sample before lipid extraction for quantification of NL, FFA and PL, respectively. After conditioning the columns with $3 \mathrm{ml}$ of hexane, the total lipid extract dissolved in $200 \mu \mathrm{l}$ of chloroform was loaded. After the chloroform was pulled through, the NL fraction was eluted with $1.8 \mathrm{ml}$ of chloroform-2-propanol (2:1, v:v) (Biosolve). The column was then loaded with $2.4 \mathrm{ml}$ of diethyl ether-acetic acid (98:2, v:v) (Biosolve) followed by $1.8 \mathrm{ml}$ of methanol (Biosolve) to elute the NEFA and PL fractions, sequentially. Each fraction was then dried under nitrogen and methylated as described previously. The FAME were then extracted by $2 \mathrm{ml}$ of hexane and separated by GC.

\section{Identification and quantification of fatty acids by GC-MS and HPLC}

Some lipid extracts were analysed by GC-MS in order to identify the CLA and CLnA accumulated by Caco- 2 cells. The preparation of the samples was performed as described previously and the fatty acid profile was obtained with a GC Trace gas chromatograph equipped with an RT2560 capillary column, a Combi PAL autosampler (CTC analystics) and an MS detector DSQ (Thermo Finnigan). The carrier gas used was He at a constant flow rate of $1 \mathrm{ml} / \mathrm{min}$ and the temperature programming was the same as the one described previously. The MS transfer line was maintained at a temperature of $250^{\circ} \mathrm{C}$. GC-MS was carried out using $70 \mathrm{eV}$ electron impact and the data were evaluated using total ion count for compound identification.

The lipid extracts analysed by GC-MS were also analysed by HPLC in order to confirm the identification. HPLC analysis was performed using an HPLC system consisting of a 307pump, a manual injector and a UV-Vis 151 detector (Gilson). Following this, three columns of Chromspher Lipids impregnated with silver $(25 \mathrm{~cm} \times 4.6 \mathrm{~mm}$ internal diameter, $5 \mu \mathrm{m}$ particle size (Chrompack, Varian)) were used in series and the oven (Jones Chromatography) was maintained at a constant temperature of $25^{\circ} \mathrm{C}$. A $5 \mu$ l sample was manually injected onto the column. The mobile phase was hexaneacetonitrile-diethyl ether-2-propanol $(99 \cdot 35: 0 \cdot 1: 0 \cdot 5: 0 \cdot 05$; by vol.) (Sigma-Aldrich and Merck) and the flow rate was $1 \mathrm{ml} /$ min. Detection of FAME with conjugated diene (CLA) was at $233 \mathrm{~nm}$ and of FAME with conjugated triene (CLnA) was at $275 \mathrm{~nm}$. The HPLC system was connected to a computer with a ChromQuest (version 2.51) software.

Standards of methylated $c 9 t 11$-CLA and $t 9 t 11$-CLA (Larodan) were used for the quantification of these two CLA. For CLnA, only a standard of methylated $\alpha$-ESA (Cayman Chemical) was available and quantification of the four CLnA ( $\alpha$-ESA, PA, $\beta$-ESA and CA) was performed on the basis of that standard.

\section{Protein determination}

After division of the cell suspension in two parts and centrifugation for $10 \mathrm{~min}$ at $500 \mathrm{~g}$ (see previous subsection), the second part was lysed in Reporter Lysis Buffer (Promega). Protein concentration was then determined using the bicinchoninic acid protein assay kit (Sigma-Aldrich), which is based on the alkaline reduction of $\mathrm{Cu}^{2+}$ to $\mathrm{Cu}^{1+}$ by proteins followed by the formation of a purple complex of $\mathrm{Cu}^{1+}$ with bicinchoninic acid. Then, $25 \mu \mathrm{l}$ of cell lysate were mixed with $200 \mu \mathrm{l}$ bicinchoninic acid working reagent. After incubation at $37^{\circ} \mathrm{C}$ for $30 \mathrm{~min}$, the $\mathrm{Cu}^{1+}$-bicinchoninic acid complex formed was quantified at $562 \mathrm{~nm}$ with a spectrophotometer (SpectraCount $^{\mathrm{TM}}$, Packard). Protein concentrations were calculated using a bovine serum albumin (Sigma-Aldrich) calibration curve obtained for each assay with a known concentration range of bovine serum albumin $(0 \cdot 2-2 \mathrm{mg} / \mathrm{ml})$.

\section{Statistical analysis}

Data for intracellular fatty acid composition and for the conversion rate are reported as means from three independent experiments, each being made of triplicates. Data were subjected to one-way ANOVA using SAS (version 9.2; SAS Institute, Inc.) coupled with the Tukey's test in order to identify means with significant differences $(P<0 \cdot 05)$.

\section{Results}

\section{Cell fatty acid composition}

The fatty acid profile of Caco- 2 cells in control conditions and upon exposure to exogenous CLnA isomers ( $\alpha$-ESA, PA, $\beta$-ESA or CA) is presented in Table 1. Statistical analysis revealed that there are no differences between the different culture conditions (control or with one of the added CLnA) when fatty acids are considered one by one, except for the CLA and CLnA. The lipid profile of these cells was relatively simple, 
Table 1. Total fatty acid composition of Caco-2 cells cultivated in serum-free medium during $7 \mathrm{~d}$ post-confluence and then incubated during $24 \mathrm{~h}$ in the presence of one exogenous conjugated linolenic acid (CLnA) isomer at $20 \mu \mathrm{mol} / \mathrm{l}$ (Mean values and standard deviations, three independent experiments)*

\begin{tabular}{|c|c|c|c|c|c|c|c|c|c|c|}
\hline \multirow[b]{2}{*}{ Fatty acids } & \multicolumn{2}{|c|}{ Control (ng/ug) } & \multicolumn{2}{|c|}{$\alpha-E S A \dagger(n g / \mu g)$} & \multicolumn{2}{|c|}{ PA $\ddagger(n g / \mu g)$} & \multicolumn{2}{|c|}{$\beta$-ESA§ (ng/ $/ \mu \mathrm{g})$} & \multicolumn{2}{|c|}{$\mathrm{CA} \|(\mathrm{ng} / \mu \mathrm{g})$} \\
\hline & Mean & SD & Mean & SD & Mean & SD & Mean & SD & Mean & SD \\
\hline $14: 0$ & $13 \cdot 5^{\mathrm{a}}$ & $2 \cdot 2$ & $14 \cdot 7^{\mathrm{a}}$ & $2 \cdot 4$ & $15 \cdot 7^{a}$ & $3 \cdot 3$ & $16 \cdot 1^{\mathrm{a}}$ & 3.3 & $16 \cdot 1^{a}$ & $2 \cdot 9$ \\
\hline $16: 0$ & $49 \cdot 3^{a}$ & $10 \cdot 2$ & $53 \cdot 4^{\mathrm{a}}$ & 9.1 & $56 \cdot 8^{a}$ & $12 \cdot 7$ & $56 \cdot 3^{a}$ & $9 \cdot 4$ & $56 \cdot 0^{\mathrm{a}}$ & 8.5 \\
\hline $18: 0$ & $20 \cdot 1^{\mathrm{a}}$ & 4.0 & $19 \cdot 9^{a}$ & 3.0 & $21 \cdot 8^{\mathrm{a}}$ & 4.5 & $20 \cdot 7^{\mathrm{a}}$ & 2.9 & $20 \cdot 2^{\mathrm{a}}$ & $3 \cdot 1$ \\
\hline $16: 1 n-7$ & $43 \cdot 1^{a}$ & 9.7 & $47 \cdot 7^{a}$ & 10.9 & $43 \cdot 7^{a}$ & $16 \cdot 1$ & $55 \cdot 4^{a}$ & $10 \cdot 9$ & $50 \cdot 5^{a}$ & $6 \cdot 0$ \\
\hline $18: 1 n-9$ & $71 \cdot 8^{a}$ & $16 \cdot 4$ & $73 \cdot 7^{a}$ & $14 \cdot 0$ & $81 \cdot 1^{a}$ & $19 \cdot 6$ & $83 \cdot 2^{\mathrm{a}}$ & $15 \cdot 7$ & $81 \cdot 3^{a}$ & $15 \cdot 0$ \\
\hline $18: 1 n-7$ & $41 \cdot 4^{\mathrm{a}}$ & 8.8 & $41 \cdot 4^{\mathrm{a}}$ & $7 \cdot 4$ & $45 \cdot 7^{a}$ & 10.5 & $46 \cdot 7^{\mathrm{a}}$ & $8 \cdot 7$ & $44 \cdot 6^{\mathrm{a}}$ & $7 \cdot 3$ \\
\hline $18: 2 n-6$ & $0 \cdot 1^{\mathrm{a}}$ & 0.1 & $0.2^{\mathrm{a}}$ & 0.1 & $0.1^{a}$ & 0.1 & $0.2^{\mathrm{a}}$ & 0.2 & $0.2^{\mathrm{a}}$ & 0.2 \\
\hline $18: 3 c 9 t 11 t 13$ & \multicolumn{2}{|c|}{$N D^{b}$} & $5 \cdot 2^{\mathrm{a}}$ & 0.5 & \multicolumn{2}{|c|}{$N D^{b}$} & \multicolumn{2}{|c|}{$N D^{b}$} & \multicolumn{2}{|c|}{$N D^{b}$} \\
\hline $18: 3 c 9 t 11 c 13$ & \multicolumn{2}{|c|}{$N D^{b}$} & \multicolumn{2}{|c|}{$N D^{\mathrm{b}}$} & $12 \cdot 1^{\mathrm{a}}$ & $3 \cdot 3$ & \multicolumn{2}{|c|}{$N D^{b}$} & \multicolumn{2}{|c|}{$N D^{b}$} \\
\hline $18: 3 t 9 t 11 t 13$ & \multicolumn{2}{|c|}{$N D^{b}$} & \multicolumn{2}{|c|}{$N D^{b}$} & \multicolumn{2}{|c|}{$N D^{b}$} & $4 \cdot 2^{a}$ & $1 \cdot 1$ & \multicolumn{2}{|c|}{$N D^{b}$} \\
\hline $18: 3 t 9 t 11 c 13$ & \multicolumn{2}{|c|}{$N D^{b}$} & & & \multicolumn{2}{|c|}{$N D^{b}$} & \multicolumn{2}{|c|}{$N D^{b}$} & $6 \cdot 4^{a}$ & $1 \cdot 1$ \\
\hline $18: 2 c 9 t 11$ & \multirow{2}{*}{\multicolumn{2}{|c|}{$\begin{array}{l}N^{c} \\
N D^{c}\end{array}$}} & $3 \cdot 0^{\mathrm{a}}$ & 0.5 & $1.4^{\mathrm{b}}$ & 0.3 & & & \multicolumn{2}{|c|}{$N D^{c}$} \\
\hline $18: 2$ t9t11 & & & & & \multicolumn{2}{|c|}{$N D^{c}$} & $5 \cdot 4^{a}$ & 1.5 & $3.9^{b}$ & 1.1 \\
\hline
\end{tabular}

$\alpha$-ESA, $\alpha$-eleostearic acid; PA, punicic acid; $\beta$-ESA, $\beta$-eleostearic acid; CA, catalpic acid; ND, not detected.

$\mathrm{a}, \mathrm{b}, \mathrm{c}$ Mean values within a row with unlike superscript letters were significantly different $(P<0.05)$.

* Each being made of triplicates, and is expressed in ng of each fatty acid per $\mu \mathrm{g}$ of cell protein.

† 9 t11t13-CLnA.

$\ddagger c 9 t 11 c 13-C L n A$.

$\S t 9 t 11 t 13-C L n A$.

|| t9t11c13-CLnA.

thanks to their cultivation in the serum-free medium. This medium is totally free of fatty acids with the exception of trace amounts of linoleic acid (18:2 n-6), added in its formulation as a complex to albumin. In these conditions, the cells were totally free of $n-6$ and $n-3$ fatty acids except for linoleic acid, which accumulated as a result of its presence in the medium. All the SFA and MUFA were thus de novo synthesised by the cells. Furthermore, the cells incubated with one of the four CLnA isomers accumulated that isomer. The GC analyses also indicated the presence of some isomers of CLA in these cells, while control cells were totally free of CLA. This indicates an efficient metabolic processing of CLnA into CLA in the Caco- 2 cells. To identify the CLA isomer produced from CLnA, further HPLC and GC-MS analyses of FAME were performed. These analyses confirmed that the isomer produced in Caco- 2 cells from $\alpha$-ESA and PA was c9t11-CLA and that the one produced from $\beta$-ESA and CA was t9t11-CLA (A-C Schneider and E Mignolet, unpublished results).

The cellular level of the accumulated -9,-11,-13 CLnA seemed similar for the isomers analysed, except for PA for which the accumulation level was almost doubled as compared to the others (Table 1). Regarding the CLA isomers produced from CLnA, their cellular levels were highly variable between the conditions, with a minimal value for cells incubated with PA $(1.4 \mathrm{ng} / \mu \mathrm{g}$ cell protein) and a maximal value for cells incubated with $\beta$-ESA $(5.4 \mathrm{ng} / \mu \mathrm{g}$ cell protein). The conversion rate, defined as the cellular level of the CLA isomer divided by the sum of the cellular levels of the CLA isomer and the CLnA isomer, of -9,-11,-13 CLnA isomer into the corresponding CLA isomer, varied significantly according to the added CLnA and ranged from about $11 \%$ for PA up to about $56 \%$ for $\beta$-ESA (Fig. 2). The differences observed may be due to the cis or trans configuration of both $\Delta-9$ and
$\Delta-13$ double bonds. Indeed, when the isomers with the same configuration of the $\Delta-9$ double bond were compared $(\alpha$-ESA $v$. PA and $\beta$-ESA $v$. CA), the conversion rate was lower when the $\Delta-13$ double bond was in its cis configuration. Conversely, when the isomers with the same configuration of the $\Delta-13$ double bond were compared ( $\alpha$-ESA $v$. $\beta$-ESA and PA $v$. CA), the conversion rate was lower when the $\Delta-9$ double bond was in its cis configuration. As a result, the conversion of PA appeared to be the lowest.

The time course of $\beta$-ESA and CA accumulation and metabolic processing into CLA in Caco-2 cells was determined in the same experimental conditions as described previously (Fig. 3). The cellular level of $\beta$-ESA appeared to increase

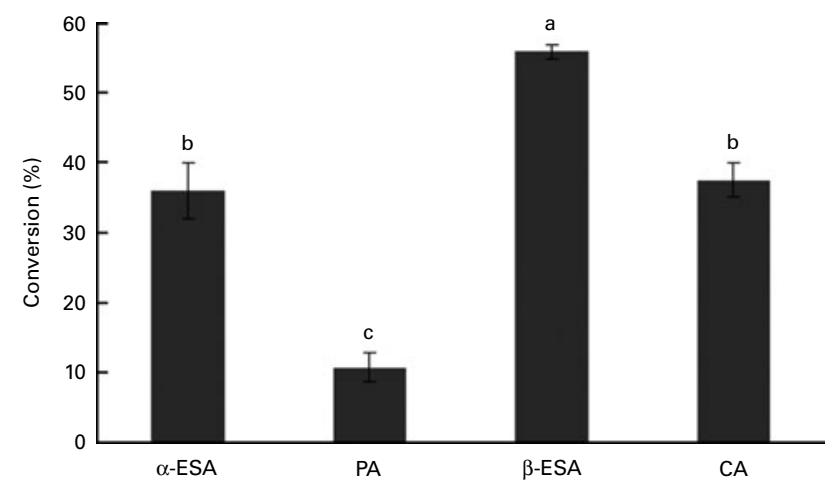

Fig. 2. Percentage of conversion of $\alpha$-eleostearic acid $(\alpha-E S A)$ and punicic acid (PA) into cis-9, trans-11-conjugated linoleic acids (CLA) and of $\beta$-eleostearic acid ( $\beta$-ESA) and catalpic acid (CA) into trans-9, trans-11-CLA upon cultivation of Caco-2 cells in serum-free medium during $7 \mathrm{~d}$ post-confluence followed by an incubation in the presence of one exogenous conjugated linolenic acid isomer during $24 \mathrm{~h}$ at $20 \mu \mathrm{mol} / \mathrm{l}$. Values are means from three independent experiments, each being made of triplicates, with standard deviations represented by vertical bars. ${ }^{a, b, c}$ Mean values with unlike letters were significantly different $(P<0.05)$. 

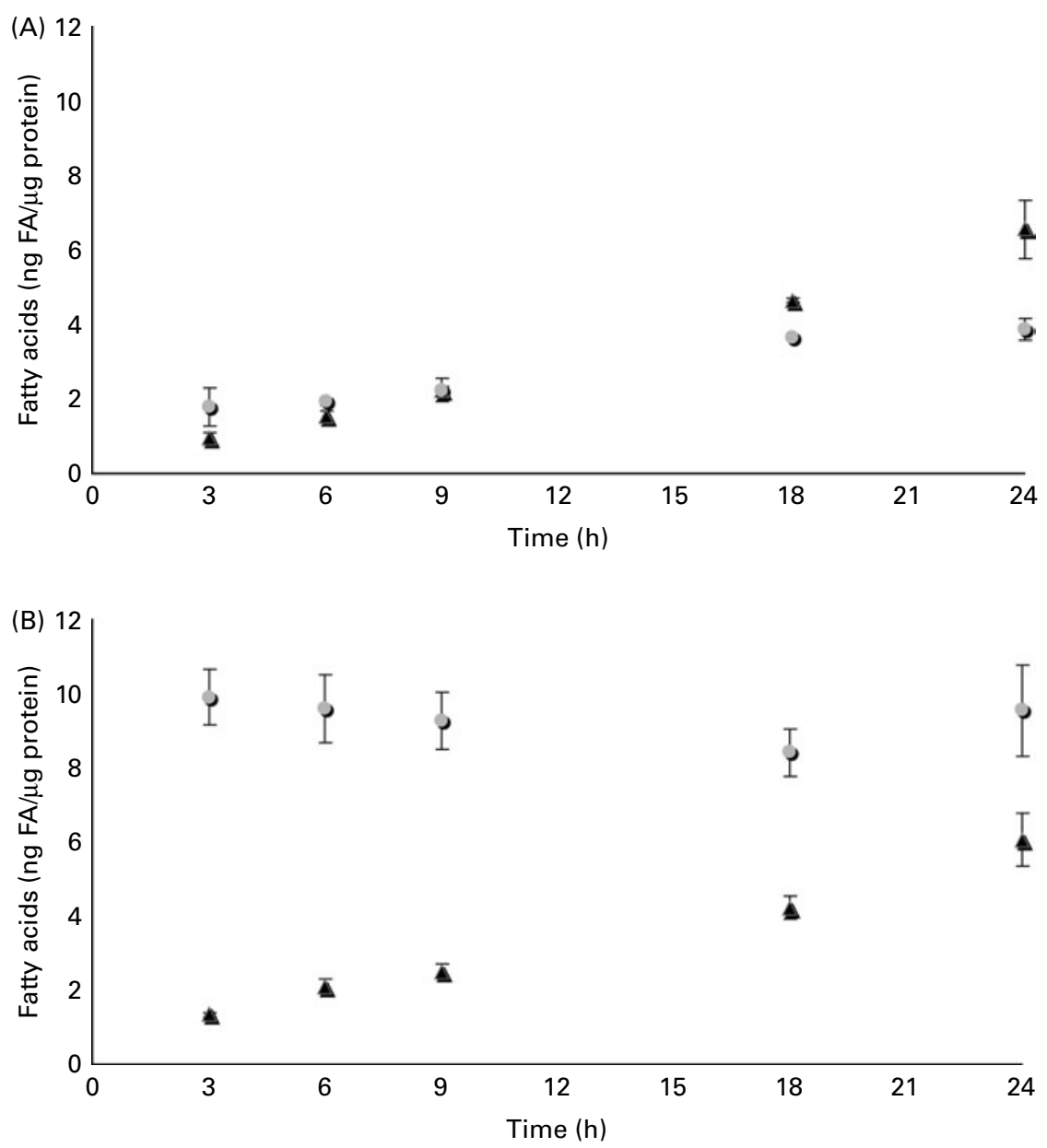

Fig. 3. Time course of accumulation and processing of $(A) \beta$-eleostearic acid ( $\beta$-ESA, $\bullet$ ) and (B) catalpic acid (CA, $\bullet)$ in Caco-2 cells cultivated in serum-free medium during $7 \mathrm{~d}$ post-confluence and then incubated with this fatty acid during $3,6,9,18$ and $24 \mathrm{~h}$ at $20 \mu \mathrm{mol} / \mathrm{l}$. Values are means in ng of fatty acids (FA) per $\mu \mathrm{g}$ of cell protein, each value being made of triplicates, with standard deviations represented by vertical bars. $\mathbf{\Lambda}$, Trans-9, trans-11-conjugated linoleic acids.

progressively during the duration of the experiment even though a plateau seemed to be reached after $18 \mathrm{~h}$. In contrast, the CA accumulation level was already at its maximum after $3 \mathrm{~h}$ of incubation and remained roughly stable until $24 \mathrm{~h}$. The cellular level of the $19 t 11$-CLA produced from $\beta$-ESA and from CA increased with time up to $24 \mathrm{~h}$ of incubation in both cases. Both accumulation kinetics could be approximated by a linear regression $\left(R^{2}\right.$ of 0.996 and 0.942 , respectively, for the $\beta$-ESA and CA conditions), which indicates a constant accumulation rate throughout the $1 \mathrm{~d}$ incubation. Furthermore, these accumulation rates were almost equivalent, as indicated by the values of the slopes of these regressions $(y=0.266$ and $y=0.254$, respectively, for the $\beta$-ESA and CA conditions). We may approximate the rate of uptake of $\beta$ ESA and CA by summing up the CLnA and CLA levels in each case, although this does not take into account possible other transformations into other metabolites as well as use for energetic purposes. This approximation suggests that Caco- 2 cells take up $\beta$-ESA relatively slowly and linearly with time, while they take up CA very quickly after administration before allowing further weak accumulation during the incubation.

\section{Distribution into lipid classes}

The distribution of the CLnA accumulated by the Caco- 2 cells, as well as that of their bioconversion products, $c 9 t 11$-CLA and t9t11-CLA, into the different lipid classes (NL, FFA and PL) was also determined (Fig. 4). It was first observed that all the fatty acids, including the CLnA or CLA isomers, were only distributed between the NL and PL fractions, since no fatty acid could be detected in the FFA fraction. Considering the CLnA isomers, our results showed that $\beta$-ESA, with its three trans double bonds, exclusively accumulated in the NL fraction. By contrast, CLnA isomers with two trans double bonds, namely $\alpha$-ESA and CA, were found at about $25 \%$ of their accumulation level in the PL fraction. Finally, as much as $60 \%$ of PA, which is an isomer with only one trans double bond, accumulated in the PL fraction. The repartition of CLA isomers indicated that the c9t11-CLA and the t9t11-CLA were more accumulated in the PL fraction than in the NL fraction, whatever the CLnA from which they were originated. Moreover, the proportion in the PL fraction appeared to be somewhat higher for the $c 9 t 11$-CLA than for the $t 9 t 11$-CLA. Indeed, when the cells were incubated with $\alpha$-ESA or PA, $67 \%$ and 
(A)

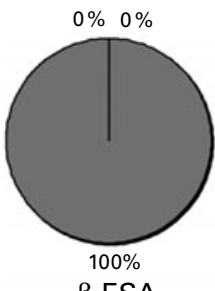

$\beta$-ESA

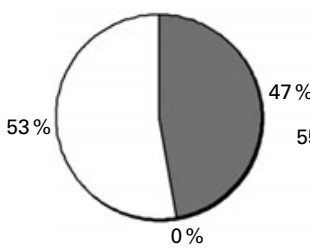

t9t11-CLA
(B)

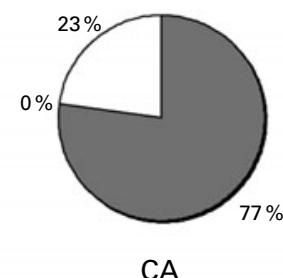

CA

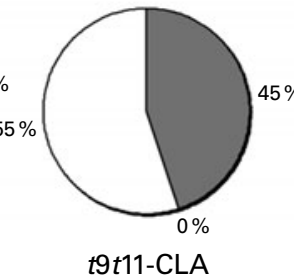

(C)

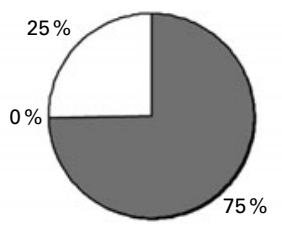

$\alpha-E S A$

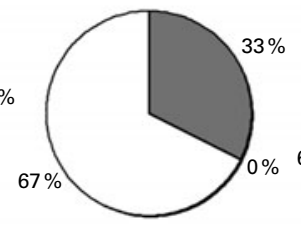

c9t11-CLA
(D)

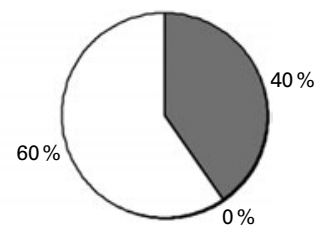

PA

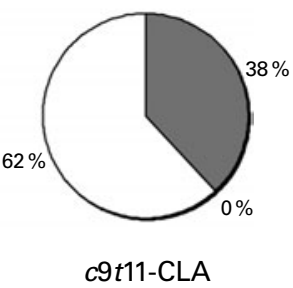

Fig. 4. Incorporation of (A) $\beta$-eleostearic acid ( $\beta$-ESA), (B) catalpic acid (CA), (C) $\alpha$-eleostearic acid ( $\alpha$-ESA) and (D) punicic acid (PA) and their metabolites (trans-9, trans-11-conjugated linoleic acids (t9t11-CLA) and cis-9, trans-11 (c9t11)-CLA) into different lipid fractions (neutral lipids ( $\square$ ), NEFA ( $\square$ ) and phospholipids $(\square)$ ) prepared from Caco- 2 cells cultivated in serum-free medium during $7 \mathrm{~d}$ post-confluence and then incubated with one of these fatty acids during $24 \mathrm{~h}$ at $20 \mu \mathrm{mol} / \mathrm{l}$. Values are means from three independent experiments.

$62 \%$ of the c9t11-CLA were, respectively, accumulated in the PL fraction, while only $53 \%$ and $55 \%$ of the $t 9 t 11$-CLA were found in this fraction, when the cells were incubated with $\beta$-ESA and CA, respectively.

\section{Discussion}

CLA have shown potential health-promoting properties ${ }^{(4-10)}$. The bulk of CLA is provided to human consumers by the diet, notably through their presence in dairy products and ruminant meat ${ }^{(2,11)}$, but dietary intake remains relatively low because CLA represent less than $1 \%$ of total ruminant lipids. In contrast, CLnA are present in high amounts in oil from several seeds, such as those from pomegranate, bitter gourd, tung or catalpa ${ }^{(14)}$. These conjugated fatty acids have thus a dietary origin and are thus taken per os. Studying their metabolism and effects in the intestinal mucosa is therefore of utmost importance.

In the present study, we provide evidence that the four -9 , $-11,-13$ isomers of CLnA can be taken up and converted into CLA in Caco-2 cells. We first confirmed that the isomer of CLA produced from $\alpha$-ESA and PA was $c 9 t 11$-CLA and that the isomer produced from $\beta$-ESA and CA was t9t11-CLA. Tsuzuki et al. ${ }^{(24,25)}$ have previously shown that the administration of tung oil, rich in $\alpha$-ESA, or pomegranate seed oil, rich in PA, leads to an accumulation of $\alpha$-ESA or PA and of $c 9 t 11$-CLA in the liver, kidney and small intestine of rats. This conversion of $\alpha$-ESA or PA into c9t11-CLA is confirmed by some studies in mice fed with a supplementation in pomegranate or bitter gourd seed oils ${ }^{(15,17,26,27)}$. Furthermore, $c 9 t 11$-CLA could be detected in plasma and erythrocyte membranes of healthy young humans supplemented with Trichosanthes kirilowi seed kernels, rich in PA, during $28 \mathrm{~d}^{(28)}$. Suzuki et al. ${ }^{(20)}$ have also shown that the administration of catalpa seed oil, containing more than $40 \%$ of CA, to rats induces an accumulation of t9t11-CLA in the liver tissue and colonic mucosa. Our study has also shown that the accumulation of the different
CLnA isomers in Caco-2 cells is quite similar, except for PA that accumulates significantly more than the three other CLnA. This difference may be due to the fact that this isomer of CLnA possesses only one trans double bond, contrary to the other ones, which possess two or three trans double bonds.

To our knowledge, this is the first time that the conversion of these four CLnA has been reported in the same model and that a comparison between the metabolism of these fatty acids is made. In addition to the effective conversion of isomers of CLnA with a trans $\Delta 13$ double bond ( $\alpha$-ESA and $\beta$-ESA) or with a cis $\Delta 13$ double bond (PA and CA) into CLA, we have further shown that the conversion rate differs according to the isomer. This appears to result partly from the cis or trans configuration of the $\Delta 13$ double bond. Indeed, if we compare the isomers of CLnA metabolised into a same isomer of CLA, $\alpha$-ESA is more effectively saturated than PA and $\beta$-ESA than CA. Such an observation has been made in mice and in rat intestine for $\alpha$-ESA and $\mathrm{PA}^{(25,26)}$ but this was never reported for $\beta$-ESA and CA. Here, we thus show that the difference in the efficiency of conversion according to the configuration of the $\Delta 13$ double bond is also applicable to CLnA metabolised into t9t11-CLA, and is probably due to a higher affinity of the enzyme for the $\Delta 13$ double bond in its trans configuration. Our results have also shown that the efficiency of the conversion into t9t11-CLA is more effective than into $c 9 t 11$-CLA. This is the reason why we realised the time course of uptake and metabolisation of $\beta$-ESA and CA, both metabolised into $19 t 11$-CLA and differing by the configuration of the $\Delta 13$ double bond. We aimed at comparing the rate of uptake of these two fatty acids in Caco-2 cells as well as the evolution of their accumulation product, t9t11-CLA. The uptake of these two CLnA in Caco-2 cells is very different. For $\beta$-ESA, the amount taken up appeared to increase progressively with time before reaching a plateau visible from $18 \mathrm{~h}$ onwards. At the other end, the cellular level of CA had already reached its maximum after $3 \mathrm{~h}$ of incubation and remained then roughly stable until $24 \mathrm{~h}$. The rate of uptake 
of CLnA could be linked to their structure, the only difference between $\beta$-ESA and CA being the geometrical structure of the $\Delta 13$ double bond. In contrast, the cellular level of t9t11-CLA produced from the metabolism of either $\beta$-ESA or CA increased with the duration of incubation in both cases. Furthermore, the rate of $t 9 t 11$-CLA accumulation was closely similar whatever the CLnA added. In our case, the production and accumulation of $t 9 t 11$-CLA in Caco- 2 cells seem thus to be independent of the CLnA from which it originates. Interestingly, Tsuzuki et al. ${ }^{(24)}$ have shown that administration of tung oil, rich in $\alpha$-ESA, to rats induced a time-dependent increase of $c 9 t 11-C L A$. In parallel, they also showed that $\alpha$-ESA concentration increased until $6 \mathrm{~h}$ after administration and had decreased by $24 \mathrm{~h}$ after administration in the plasma, liver, kidney and small intestine mucosa. Additionally, Yuan et al. ${ }^{(39)}$ have shown that the concentration of PA in the plasma, liver, heart and adipose tissue in rats fed with T. kirilowii seed oil, rich in PA, increased until $12 \mathrm{~h}$ after treatment and then decreased after $24 \mathrm{~h}$. They have also reported that the accumulation of c9t11-CLA varied according to the tissue analysed. For example, it increased with time up to $24 \mathrm{~h}$ in the brain and kidney or reached a peak at $12 \mathrm{~h}$ and then decreased in the liver, heart and adipose tissue.

In order to improve our knowledge of the metabolism of CLnA, the distribution of these fatty acids as well as of their metabolites between NL, FFA and PL fractions was also studied. We first observed that the added CLnA as well as the isomers of CLA produced by Caco- 2 cells were distributed only between the NL and PL fractions from cells. Furthermore, the distribution of the four CLnA isomers between the NL and PL fractions was correlated to the number of trans double bonds. Indeed, the CLnA with three trans double bonds ( $\beta$-ESA) accumulated only in the NL fraction, while those with two trans double bonds ( $\alpha$-ESA and CA) accumulated for about $75 \%$ in the NL fraction and for about $25 \%$ in the PL fraction; and finally, the isomer with only one trans double bond (PA) accumulated for about $60 \%$ in the PL fraction. So, the more the fatty acid possesses trans double bonds, the more it accumulates in the NL fraction in comparison to the PL fraction. Finally, the position of the trans double bond in the CLnA seemed without influence on the repartition between the NL and PL fractions. Indeed, $\alpha$-ESA and CA were identically distributed between these two fractions whereas their trans double bonds were located at different positions: 11 and 13 for $\alpha$-ESA and 9 and 11 for CA.

For the CLA produced, we can observe that the distribution in the two fractions (NL and PL) seems to be also related to the number of trans double bonds but to a lesser extent than for CLnA. Indeed, t9t11-CLA appeared to be somewhat more accumulated in the NL fraction in comparison with $c 9 t 11$ CLA. Moreover, the repartition of the two CLA isomers is governed by their structure rather than by the CLnA from which they originate. For a same isomer of CLA, the distribution between NL and PL was indeed very similar in spite of the distribution of its precursor CLnA.

The formation of $c 9 t 11$-CLA or $t 9 t 11$-CLA from the four $-9,-11,-13$ isomers of CLnA results from a reduction of the double bond in the $\Delta 13$ position but at the present time, the mechanism of this conversion remains unclear. Tsuzuki et al. ${ }^{(24,25)}$ showed that $\alpha$-ESA and PA were saturated at the $\Delta 13$ position by an NADPH-dependent enzyme and converted into $c 9 t 11$-CLA. They speculated that this $\Delta 13$ saturation was carried out either by an unknown enzyme recognising conjugated trienoic acids or by the enzyme active in the leukotriene B4 reductive pathway, LTB4 12-HD also named PGR. Moreover, Yuan et al. ${ }^{(26)}$ mentioned that a retinol saturase was recently identified by Moise et al. ${ }^{(40,41)}$ in zebrafish and mice. This enzyme carries out the saturation of the $\Delta 13$ double bond of the all-trans-retinol to produce alltrans-13,14-dihydroretinol. Furthermore, isomers other than $-9,-11,-13$ CLnA share the conjugated double bond system of c9t11-CLA, like c9t11c15-CLnA. Plourde et al. ${ }^{(42)}$ showed that administration of $c 9 t 11 c 15$-CLnA to rats induced its accumulation in the liver, plasma and adipose tissue while $c 9 t 11$-CLA did not. These results combined with ours suggest that all CLnA containing the $c 9 t 11$ structure cannot be reduced into $c 9 t 11-C L A$ and that a specific enzyme acts specifically on the $\Delta 13$ double bond. More studies are needed to identify the active enzyme and understand its mechanism of action.

In conclusion, the present study provides strong evidence that human enterocytes represented here by Caco- 2 cells, which although from cancerous origin, are probably the best in vitro model for these cells, are of major importance in the metabolism of CLnA. These cells are indeed exposed to bolus, are the major site for fatty acid uptake and export nutrients to other organs via the blood or lymphatic circulation. We show here that they are also able to reduce CLnA into CLA. The repartition into the different lipid classes shows that enterocytes store a significant part of these CLnA in the NL fraction, suggesting that CLnA and their metabolites should be exported to other organs. All these observations have gained importance since it is known that CLA, and principally c9t11-CLA, have many biological activities ${ }^{(4-10)}$. The consumption of CLnA could thus become an interesting approach for people with an insufficient intake in CLA.

\section{Acknowledgements}

The present work was supported by grants from the Fonds de la Soutein de la Recherche (FSR) of the Université catholique de Louvain A.-C. S. is a fellow of the Fonds de la Recherche Scientifique (FRIA) of the Communauté française de Belgique. A.-C. S., Y.-J. S. and Y. L. designed the research; A.-C. S. conducted the research; A.-C. S. and E. M. analysed the data; A.-C. S. wrote the paper. Y. L. had primary responsibility for the final content. All authors read and approved the final manuscript and have no conflicts of interest.

\section{References}

1. Ha YL, Grimm NK \& Pariza MW (1987) Anticarcinogens from fried ground beef: heat-altered derivatives of linoleic acid. Carcinogenesis 8, 1881-1887.

2. Pariza MW, Park Y \& Cook ME (2000) Mechanisms of action of conjugated linoleic acid: evidence and speculation. Proc Soc Exp Biol Med 223, 8-13.

3. Iwabuchi M, Kohno-Murase J \& Imamura J (2003) Delta 12-oleate desaturase-related enzymes associated with formation 
of conjugated trans-delta11, cis-delta13 double bounds J Biol Chem 278, 4603-4610.

4. Ip C, Chin SF, Scimeca JA, et al. (1991) Mammary cancer prevention by conjugated dienoic derivative of linoleic acid. Cancer Res 51, 6118-6124.

5. Belury MA (2002) Inhibition of carcinogenesis by conjugated linoleic acid: potential mechanisms of action. J Nutr $\mathbf{1 3 2}$, 2995-2998.

6. McLeod RS, LeBlanc AM, Langille MA, et al. (2004) Conjugated linoleic acids, atherosclerosis and hepatic very-low-density lipoprotein metabolism. Am J Clin Nutr 79, 1169S-1174S.

7. Kritchevsky D, Tepper SA, Wright S, et al. (2004) Conjugated linoleic acid isomer effects in atherosclerosis: growth and regression of lesions. Lipids 39, 611-616.

8. Bassaganya-Riera J, Hontecillas R \& Beitz DC (2002) Colonic anti-inflammatory mechanisms of conjugated linoleic acid. Clin Nutr 21, 451-459.

9. O'Shea M, Bassaganya-Riera J \& Mohede ICM (2004) Immunomodulatory properties of conjugated linoleic acid. Am J Clin Nutr 79, 1199S-1206S.

10. Reynolds CM, Loscher CE, Moloney AP, et al. (2008) Cis-9, trans-11-conjugated linoleic acid but not its precursor transvaccenic acid attenuate inflammatory markers in the human colonic epithelial cell line Caco-2. BrJ Nutr 100, 13-17.

11. Badinga L \& Greene ES (2006) Physiological properties of conjugated linoleic acid and implications for human health. Nutr Clin Pract 21, 367-373.

12. MacDonald HB (2000) Conjugated linoleic acid and disease prevention: a review of current knowledge. J Am Coll Nutr 19, 111S-118S.

13. Takagi T \& Itabashi Y (1981) Occurrence of mixtures of geometrical isomers of conjugated octadecatrienoic acids in some seed oils: analysis by open-tubular gas liquid chromatography and high performance liquid chromatography. Lipids 16, 546-551.

14. Hennessy AA, Ross RP, Devery R, et al. (2011) The health promoting properties of the conjugated isomers of $\alpha$-linolenic acid. Lipids 46, 105-119.

15. Yamasaki M, Kitagawa T, Koyanagi N, et al. (2006) Dietary effect of pomegranate seed oil on immune function and lipid metabolism in mice. Nutrition 22, 54-59.

16. Ike K, Uchida Y, Nakamura T, et al. (2005) Induction of interferon-gamma (IFN-gamma) and $\mathrm{T}$ helper 1 (Th1) immune response by bitter gourd extract. J Vet Med Sci 67, 521-524.

17. Koba K, Imamura J, Akashoshi A, et al. (2007) Genetically modified rapeseed oil containing cis-9,trans-11,cis-13octadecatrienoic acid affects body fat mass and lipid metabolism in mice. J Agric Food Chem 55, 3741-3748.

18. Bassaganya-Riera J, Guri AM \& Hontecillas R (2011) Treatment of obesity-related complications with novel classes of naturally occurring PPAR agonists. J Obes 2011, 897-894.

19. Kohno H, Suzuki R, Yasui Y, et al. (2004) Pomegranate seed oil rich in conjugated linolenic acid suppresses chemically induced colon carcinogenis in rats. Cancer Sci 95, 481-486.

20. Suzuki R, Yasui Y, Kohno H, et al. (2006) Catalpa seed oil rich in $9 t, 11 t, 13 c$-conjugated linolenic acid suppresses the development of colonic aberrant crypt foci induced by azoxymethane in rats. Oncol Rep 16, 989-996.

21. Kim ND, Mehta R, Yu W, et al. (2002) Chemoprotective and adjuvant therapeutic potential of pomegranate (Punica granatum) for human breast cancer. Breast Cancer Res Treat 71, 203-217.

22. Igarashi M \& Miyazawa T (2000) Newly recognized cytotoxic effect of conjugated trienoic fatty acids on cultured human tumor cells. Cancer Lett 148, 173-179.
23. Yasui Y, Hosokawa M, Sahara T, et al. (2005) Bitter gourd seed fatty acid rich in $9 c, 11 t, 13 t$-conjugated linolenic acid induces apoptosis and up-regulates the GADD45, p53 and PPAR $y$ in human colon cancer Caco-2 cells. Prostaglandins Leukot Essent Fatty Acids 73, 113-119.

24. Tsuzuki T, Tokuyama Y, Igarashi M, et al. (2004) $\alpha$-Eleostearic acid (9Z11E13E-18:3) is quickly converted to conjugated linoleic acid (9Z11E-18:2) in rats. J Nutr 134, 2634-2639.

25. Tsuzuki T, Kawakami Y, Abe R, et al. (2006) Conjugated linolenic acid is slowly absorbed in rat intestine, but quickly converted to conjugated linoleic acid. J Nutr 136, 2153-2159.

26. Yuan GF, Sinclair AJ, Zhou CQ, et al. (2009) $\alpha$-Eleostearic acid is more effectively metabolized into conjugated linoleic acid than punicic acid in mice. J Sci Food Agric 89, 1006-1011.

27. Yuan GF, Sinclair AJ, Sun HY, et al. (2009) Fatty acid composition in tissues of mice fed diets containing conjugated linolenic acid and conjugated linoleic acid. J Food Lipids 16, 148-163.

28. Yuan GF, Sinclair AJ, Xu C, et al. (2009) Incorporation and metabolism of punicic acid in healthy young humans. Mol Nutr Food Res 53, 1336-1342.

29. Yamamoto T, Yokomizo T, Nakao A, et al. (2001) Immunohistochemical localization of guinea-pig leukotriene $\mathrm{B}_{4}$ 12-hydroxydehydrogenase/15-ketoprostaglandin 13-reductase. Eur J Biochem 268, 6105-6113.

30. Tso P, Nauli A \& Lo CM (2004) Enterocyte fatty acid uptake and intestinal fatty acid-binding protein. Biochem Soc Trans 32, $75-78$.

31. Iqbal J \& Hussain MM (2009) Intestinal lipid absorption. $A m J$ Endocrinol Metabol 296, E1183-E1194.

32. Garg ML, Keelan M, Thomson AB, et al. (1988) Fatty acid desaturation in the intestinal mucosa. Biochim Biophys Acta 958, 139-141.

33. Trotter PJ, Ho SY \& Storch J (1996) Fatty acid uptake by Caco-2 human intestinal cells. J Lipid Res 37, 336-346.

34. Dias VC \& Parsons HG (1995) Modulation in delta-9, delta-6 and delta- 5 fatty acid desaturase activity in the human intestinal Caco-2 cell line. J Lipid Res 36, 552-563.

35. Huang YS, Liu JW, Koba K, et al. (1995) n-3 and n-6 fatty acid metabolism in undifferentiated and differentiated human intestine cell line (Caco-2). Mol Cell Biochem 151, 121-130.

36. Schneider YJ (1989) Optimisation of hybridoma cell growth and monoclonal antibody secretion in a chemically defined, serum- and protein-free culture medium. J Immunol Methods 116, 65-77.

37. Halleux C \& Schneider YJ (1991) Iron absorption by intestinal epithelial cells: $1 . \mathrm{CaCO}_{2}$ cells cultivated in serum-free medium, on polyethyleneterephtalate microporous membranes, as an in vitro model. In vitro Cell Dev Biol $27 \mathrm{~A}$ 293-302.

38. Bligh EG \& Dyer WJ (1959) A rapid method of total lipid extraction and purification. Can J Biochem Physiol 37, 911-917.

39. Yuan GF, Yuan JQ \& Duo L (2009) Punicic acid from Trichosanthes kirilowii seed oil is rapidely metabolized to conjugated linoleic acid in rats. J Med Food 12, 416-422.

40. Moise AR, Isken A, Dominguez M, et al. (2007) Specificity of zebrafish retinol saturase: formation of all-trans-13,14-dihydroretinol and all-trans-7,8-dihydroretinol. Biochemistry $\mathbf{4 6}$, 1811-1820.

41. Moise AR, Kuksa V, Imanishi Y, et al. (2004) Identification of all-trans-retinol:all-trans-13,14-dihydroretinol saturase. J Biol Chem 279, 50230-50242.

42. Plourde M, Sergiel JP, Chardigny JM, et al. (2006) Absorption and metabolism of conjugated $\alpha$-linolenic acid given as free fatty acids or triacylglycerols in rats. Nutr Metab 3, 8 . 